\title{
Symptomatic perianeurysmal parenchymal cyst: case illustration
}

\author{
Charles G. Kulwin, MD, Ravi H. Gandhi, MD, Neal B. Patel, MD, and Troy D. Payner, MD \\ Department of Neurosurgery, Indiana University School of Medicine and Goodman Campbell Brain and Spine, \\ Indianapolis, Indiana \\ http://thejns.org/doi/abs/10.3171/2014.9.JNS14712 \\ KEY WORDS aneurysm; perianeurysmal cyst; intraoperative angiography; intraoperative microultrasound; \\ vascular disorders
}

A 74-year-old woman presented with chronic headache and gait imbalance. Examination revealed left pronator drift and dysmetria. Contrasted MRI revealed a right frontotemporal enhancing lesion (Fig. 1A) with adjacent nonenhancing cyst (Fig. 1B). Differential diagnosis included primarily vascular or neoplastic entities; the patient declined intervention, so serial imaging was recommended. At 9 months (Fig. 1C) the cyst had enlarged. At 12 months she developed progressive left hemiparesis and was nonambulatory. Noncontrasted CT revealed further cystic enlargement; she declined further studies including vascular imaging.

Intraoperative findings included a glial cyst filled with greenish fluid and a firm, smoothwalled mass with a traversing artery (Fig. 1D). Intraoperative micro-Doppler identified arterial pulsation in the lesion; intraoperative angiography confirmed the diagnosis of distal middle cerebral artery (MCA) aneurysm. Videoangiography confirmed retrograde filling of the distal vessel and aneurysm occlusion via trapping. Postoperatively there was immediate improvement in left-sided symptoms, with return of ambulation at follow-up.

Perianeurysmal parenchymal cysts are rare, with few reported cases. ${ }^{1}$ Cyst formation may involve ischemic encephalomalacia, hemorrhage, or inflammation related to aneurysm thrombosis. ${ }^{4}$ Cyst resolution postembolization is described, ${ }^{3}$ but when symptomatic, surgical drainage remains effective. Prior descriptions report an enhancing nodule with surrounding cyst, ${ }^{1,2}$ which may be mistaken for a neoplasm. ${ }^{1}$ This is the first description of an enlarging perianeurysmal cyst without aneurysm change or hemorrhage. In the absence of preoperative vascular imaging, we demonstrate the value of intraoperative micro-Doppler as a safe and timely technique to evaluate an encapsulated lesion of concern as a possible aneurysm.

\section{References}

1. Benvenuti L, Gagliardi R, Scazzeri F, Gaglianone $\mathrm{S}$ : Parenchymal perianeurysmal cyst in the brain: case report. Neurosurgery 58:E788, 2006

2. Hirota N, Ueno J, Naitoh H, Sugiyama K, Karasawa H, Kin H, et al: Giant aneurysm associated with a large cyst. Case illustration. J Neurosurg 91:160, 1999

3. Martinez Galdamez M, Saura Lorente P, Palomera Rico A, Pérez-Higueras A: Intracranial perianeurysmal cyst: still a dilemma. A case report with endovascular management. Neuroradiol J 24:743-748, 2011

4. Sato N, Sze G, Awad IA, Putman CM, Shibazaki T, Endo K: Parenchymal perianeurysmal cystic changes in the brain: report of five cases. Radiology 215:229-233, 2000

\section{Author Contributions}

Conception and design: all authors. Acquisition of data: Patel. Analysis and interpretation of data: Kulwin, Gandhi, Patel. Drafting the article: Kulwin. Critically revising the article: all authors. Reviewed submitted version of manuscript: all authors. Study supervision: Payner. 

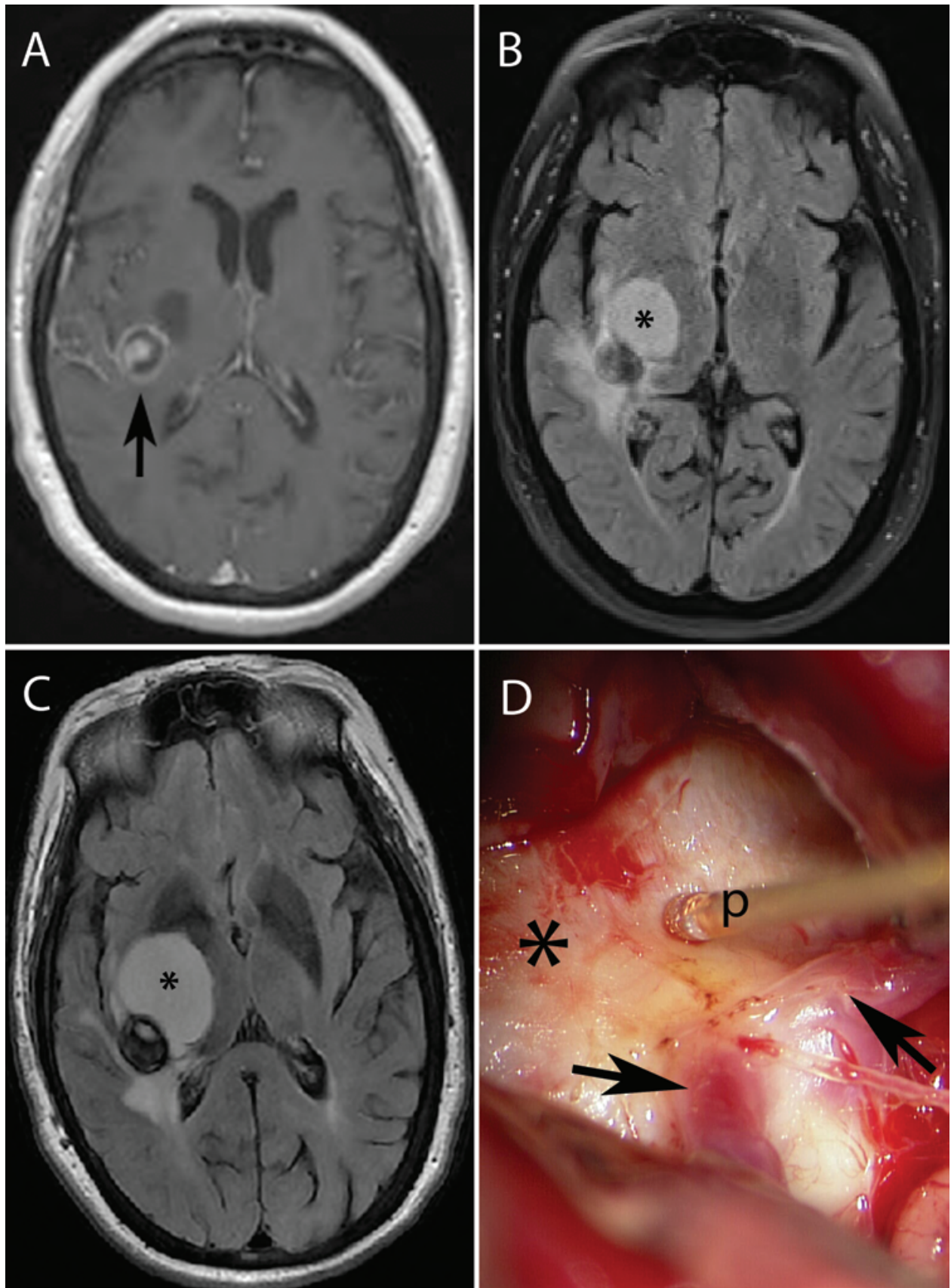

FIG. 1. Initial axial T1-weighted postcontrast (A) and T2 FLAIR (B) MR images revealing an enhancing nodule with surrounding ring enhancement (arrow) and adjacent nonenhancing cyst (asterisk) in the posterior right temporal lobe. The cyst demonstrates growth on T2 FLAIR imaging at 9-month follow-up (C). Of note, the cyst fluid differs from CSF on the FLAIR sequence. Intraoperative photograph (D) demonstrating the large smooth-walled structure (asterisk), with a distal MCA branch entering and leaving (arrows). Micro-Doppler probe (p) confirmed arterial pulsation in the suspected aneurysm. Figure is available in color online only. 